\title{
Measuring medical inflation for health insurance portfolios in Belgium
}

\author{
Jan Dhaene ${ }^{[a] *}$ \\ Hamza Hanbali ${ }^{[a] \dagger}$ \\ ${ }^{[a]}$ Research Center Insurance, AFI, Faculty of Business and Economics, \\ KU Leuven, Leuven, Belgium
}

Version: April 4, 2019

\begin{abstract}
We investigate the construction of medical inflation indexes. Such indexes can be used to update premiums of lifelong health insurance contracts. We compare the accuracy of the medical indexes currently applied in Belgium for private health insurance contracts with product-specific experience-based indexes. The latter enable to better capture product-specific systematic deviations due to medical inflation, but their application might raise some practical problems. Therefore, we propose an alternative way to construct medical inflation indexes. Several numerical examples are used to compare the performance of the newly proposed indexes with the experience-based and the current Belgian approaches. The conclusion drawn from these numerical examples is that the newly proposed indexes provide good approximations for the product-specific experience-based indexes without having their practical limitations.
\end{abstract}

Keywords: medical inflation index, lifelong health insurance, systematic risk.

\section{Introduction and some notations}

Belgian regulation allows insurers to update level premiums of lifelong private health insurance contracts using specific medical inflation indexes. These premium updates are meant to correct for unpredictable systematic changes in medical claim payments per underwritten policy. Throughout the paper, we refer to these systematic changes as medical inflation. Some obvious drivers of such an inflation are medical breakthroughs, pharmacological innovations, health workers' salaries, increasing life expectancy, global

*jan.dhaene@kuleuven.be

†hamza.hanbali@kuleuven.be 
obesity and pandemics. The medical inflation under interest here is specific to the private health insurance sector. Hence, other drivers with substantial importance can be related to the increase in service utilization and to new political decisions having an impact on the Social Security threshold above which private insurers intervene. The Belgian government provides market-wide medical indexes, which are published every year for 5 different age categories (0-19, 20-34, 35-49, 50-64 and 65+) and 4 different types of covered medical services (hospitalization with stay in a private room, hospitalization with stay in a shared room, dental care costs, ambulatory care costs).

In the present paper, we propose a methodology to construct indexes capturing the cost evolution of medical services. These indexes can be used to adapt premiums for lifelong private health insurance covers. We present and discuss the existing Belgian approach and propose an enhanced alternative. Note that medical indexes differ from consumer price indexes. Moreover, constructing them using a basket-based approach would suffer from technical limitations. One of these limitations is the fact that quantifying some of the drivers of medical inflation in the insurance sector may be not be feasible. Moreover, for the drivers that can be quantified, determining their weights in the basket can be challenging. Another limitation is that medical indexes constructed in such a manner would not be age-dependent, whereas medical inflation is likely to be age-dependence ${ }^{1}$. In order to overcome these drawbacks, Devolder et al. (2008) suggest an actuarial approach based on the claim costs experienced in the sector, which has been adopted for Belgian private health insurance. Although this method has shown its merits, the evolution of the health insurance market raises some shortcomings in both the calculation of the indexes and their application.

Concerning the calculation of the market-wide medical inflation indexes for each type of covered medical service, the current Belgian approach allocates the full data of any health insurance product to a single category, namely the type of cover which has the highest weight in the claims over the past year. This implies that the market-wide index for each type of cover might be 'polluted' by claims not corresponding to that cover.

Concerning the index that can be used to update the premiums of a particular health insurance product, the current Belgian approach consists of using the market index that corresponds to the 'most expensive' cover in that product. This means that the choice of the index for a particular product does not take into account the proportions of the costs related to the different types of covers in the portfolio.

In this paper, we will assume that the set of products as well as the set of covered medical services is time-independent over the two consecutive periods $(t-2, t-1)$ and $(t-1, t)$ that we will consider to determine the medical indexes. We denote the set of products by $\{1,2, \cdots, J\}$ and the set of types of covers by $\{1,2, \cdots, K\}$. Newly launched products or newly introduced covers require a special treatment, which will not be considered here. We will also assume that each medical service is covered by at least one product and that the types of covers included in a product do not change in the observation period.

An insurance company in the private health insurance market may sell different prod-

\footnotetext{
${ }^{1} \mathrm{We}$ refer the reader to https://statbel.fgov.be/fr/themes/prix-la-consommation/ indice-medical for the official Belgian figures.
} 
ucts, implying that $J$ is larger than or equal to the number of insurance companies in the market. Insurance products may cover one or more medical services. For instance, a product may only cover shared room hospital stays (in order to reduce the level of the premium). A product covering stays in a private room will always include a cover for stays in a shared room, as private rooms may not be available at hospitalization. Note however, that the insurer should treat the corresponding invoiced amount as if the policyholder stayed in a private room.

The medical indexes that we will investigate can be determined for different agecategories. In the following derivations, we consider a single age category, as the methodology is identical for each category.

For any product $j=1,2, \ldots, J$ and any type of cover $k=1,2, \ldots, K$, we introduce the following notations:

- $C_{j}^{(k)}(t)$ : aggregate claims resulting from cover $k$ of product $j$ in year $(t-1, t)$.

- $C_{j}(t)=\sum_{k=1}^{K} C_{j}^{(k)}(t)$ : aggregate claims of product $j$ in year $(t-1, t)$.

- $l_{j}(t)$ : total number of insureds of product $j$ in year $(t-1, t)$.

Similarly, we introduce the corresponding quantities $C_{j}^{(k)}(t-1), C_{j}(t-1)$ and $l_{j}(t-1)$, which are all related to year $(t-2, t-1)$. In practice, the method used to determine $l_{j}(t-1)$ and $l_{j}(t)$ should account for exits and entries during the year, and should be fixed in order to ensure coherence in the data collected from the different insurance companies.

We also use the notation $I^{(k)}$ for the set of products in the market which include cover $k$, i.e.

$$
I^{(k)}=\{j \in\{1,2, \ldots, J\} \mid \text { medical service } k \text { is covered by product } j\},
$$

for any $k \in\{1,2, \cdots, K\}$. The letter $I$ is chosen to indicate this set, in order to remind that the cover $k$ is 'included'. For any product $j$ in $I^{(k)}$, we will assume that $C_{j}^{(k)}(t-1)>0$ and $C_{j}^{(k)}(t)>0$. Furthermore, when $j \notin I^{(k)}$, we set $C_{j}^{(k)}(t-1)=C_{j}^{(k)}(t)=0$. Our previous assumption that each medical service is covered by at least one product implies that all the sets $I^{(k)}$ are non-empty. Finally, we will assume that $l_{j}(t-1)>0$ and $l_{j}(t)>0$.

In what follows, we will suppose that we have arrived at time $t$ and that we want to determine the increase of the medical costs from year $(t-2, t-1)$ to year $(t-1, t)$, for each type of cover and each product, as well as for all types of covers in the whole market. These observed increases can then be used as the market indexes at time $t$ to update the premiums of the products for the coming year $(t, t+1)$. In Section 2, we discuss the current Belgian method for constructing medical inflation indexes. We highlight some of its limitations and we further motivate the need of an alternative construction. In Section 3. we introduce indexes capturing the experienced medical inflation for each type of cover in each product. As it is discussed in the same section, applying these indexes might not be desirable from the viewpoint of the regulator as well as insurance companies. However, these product-specific experience-based indexes have to be introduced to describe the Belgian indexes and the proposed indexes. In Section 4, we introduce alternative medical 
inflation indexes and compare their accuracy with respect to the current Belgian ones. Finally, we conclude the paper in Section 5 and discuss some possible topics for future research.

Note that the derivations allow for two possible interpretations for the aggregate claims. The first interpretation is to consider all the $C_{j}^{(k)}$ as gross claims, which are defined as total invoiced amounts minus payments from Social Security ${ }^{2}$. The second interpretation consists in considering all $C_{j}^{(k)}$ as net claims, which correspond to the amounts effectively paid by the insurers, i.e. the gross claims minus claims not covered by the insurer. Gross and net claims will differ e.g. in case the insurer only covers a proportion of the gross claims, or in case of a maximum claim payment per hospitalization day.

\section{The official Belgian medical inflation indexes}

This section considers (a somewhat simplified version of) the current Belgian system for lifelong level premium private insurance contracts. In this system, one distinguishes 4 types of covered medical services: private room stays (or private room inpatient), shared room stays (or shared room inpatient), dental care and ambulatory care (or outpatient), meaning that $K=4$. A health insurance product may cover more than one medical service. In case a product covers more than one type of cover, current Belgian law requires to classify that product into a single category. The appointed category of a product corresponds to the type of cover with the highest weight in the overall claims of that product, where this categorization is done at product level. Therefore, product $j$ is classified as a 'type of cover $k$ ' - product in year $(t-1, t)$ if $C_{j}^{(k)}(t)$ is such that:

$$
C_{j}^{(k)}(t)=\max \left\{C_{j}^{(1)}(t), \ldots, C_{j}^{(K)}(t)\right\}
$$

For $k=1,2, \ldots, K$, the set of 'type of cover $k$ ' - products in year $(t-1, t)$ is denoted by $M^{(k)}(t)$, where the letter $M$ is chosen to remind that the claim is a maximum, i.e.

$$
M^{(k)}(t)=\left\{j \in I^{(k)} \mid j \text { is a type of cover } k \text { - product in year }(t-1, t)\right\} .
$$

The set of 'type of cover $k$ ' - products in year $(t-2, t-1)$, which is denoted by $M^{(k)}(t-1)$, is defined in a similar way.

In the current Belgian system, each $C_{j}^{(k)}(t)$ is a gross claim payment, i.e. the total amount of invoices that have been received for type of cover $k$ of product $j$ in year $(t-1, t)$, corrected for the payments made by Social Security.

Every year the medical index is determined for each type of cover $k$, based on aggregated data from the market. Determining the index for a given product is thus a two step procedure. The first step is to determine the medical inflation index at the market level. The second step is to choose the appropriate index for the product under interest. Therefore, the first step is such that at time $t$, the index for 'type of cover $k$ ' at the market

\footnotetext{
${ }^{2}$ Interested readers can find more information at https://www. socialsecurity.be/
} 
level is denoted by $i_{m}^{(k)}(t)$, where the subscript ' $m$ ' refers to the market. This index follows from the equation:

$$
\frac{\sum_{j \in M^{(k)}(t-1)} C_{j}(t-1)}{\sum_{j \in M^{(k)}(t-1)} l_{j}(t-1)} \times\left(1+i_{m}^{(k)}(t)\right)=\frac{\sum_{j \in M^{(k)}(t)} C_{j}(t)}{\sum_{j \in M^{(k)}(t)} l_{j}(t)} .
$$

Notice that the index is well-defined, provided neither $M^{(k)}(t-1)$ nor $M^{(k)}(t)$ is empty. The right hand side of definition (1) corresponds to the total amount of gross claims for 'type of cover $k$ ' - products in the market, divided by the total number of insurance contracts of 'type of cover $k$ ' - products, in the year $(t-1, t)$. On the left hand side, the same average appears for the previous year $(t-2, t-1)$. The index $i_{m}^{(k)}(t)$ can be interpreted as an estimate for the observed medical inflation of type of cover $k$ in the market, from year $(t-2, t-1)$ to $(t-1, t)$.

For the second step, Belgian law stipulates that in the coming year $(t, t+1)$, the premiums for product $j$ can be updated taking into account the index $i_{j}(t)$, which is defined by:

$$
i_{j}(t)=i_{m}^{(k)}(t), \quad \text { if } j \in M^{(k)}(t) .
$$

This means that the premiums for 'type of cover $k$ ' - product $j$ can be updated taking into account the 'type of cover $k$ ' - index $i_{m}^{(k)}(t)$. Notice that the index $i_{j}(t)$ is not defined in case $M^{(k)}(t-1)$ is empty.

Loosely speaking, the Belgian Royal Decree of 18 March 2016 regulating the application of the medical index stipulates that the maximal premium increase for lifelong contracts resulting in an actuarial reserve (with either lifelong or age-category specific level premiums) is equal to $1.5 \times i_{j}(t)$. This means that the premium of product $j$ for the period $(t, t+1)$, which is denoted by $\pi_{j}(t)$ is determined from the premium of the corresponding product for the period $(t-1, t)$ as follows:

$$
\pi_{j}(t)=\left(1+1.5 \times i_{m}^{(k)}(t)\right) \pi_{j}(t-1),
$$

if the type of cover with the highest weight in that product is $k$. The factor 1.5 is a 'rule of thumb' that is meant to restore the broken actuarial equivalence at time $t$, i.e. to ensure that after updating the premium at time $t$, the available reserve is again equal to the required reserve at that time. In other words, the effect of medical inflation on the reserves is completely paid by the policyholders by increasing of the level premiums with more than the increase suggested by the medical inflation index. We refer to Hanbali et al. (2017) for an overview of the Belgian regulation concerning the application of the medical index up to 2016. Moreover, we refer to Devolder et al. (2008) for a discussion on updating mechanisms as well as on the construction of the market medical index, and to Denuit et al. (2017) for the derivation of actuarially fair indexing mechanisms for lifelong health insurance covers with leveled premiums. An extension to contracts including withdrawal benefits is considered in Dhaene et al. (2017).

From (1), we observe that the total claim payments of 'type of cover $k$ ' - product $j$ are entirely used to calculate the market index $i_{m}^{(k)}$ for category $k$. Moreover, the premiums of 
this product can be indexed by the category $k$ index $i_{m}^{(k)}(t)$ in year $(t, t+1)$. In case each product only covers a single type of cover, the Belgian updating mechanism is definitely appropriate. In practice however, most products cover more than a single type of cover.

Private room medical costs are often increasing faster than shared room medical costs. Hence, taking into account private room costs when calculating the shared room market index leads to a value of this index which is too high for the shared room cover. Similarly, this approach will lead to a too low index for the private room cover. Moreover, dental care in a product which combines several types of covers is usually not the cover with the highest claims payments. Therefore, the dental care medical index of the market is in practice only based on the claim payments of 'pure' dental care products, ignoring dental care data coming from products with several types of covers.

Concerning the choice of the medical index that has to be applied for a particular product, a problem may arise in a multiple types of cover environment. To illustrate this point, suppose that a certain product is only covering medical services 1 and 2 (private and shared rooms), and that the proportions of the claims related to these types of cover are $49 \%$ and $51 \%$, respectively. The premiums of this product can then be indexed using the market index $i_{m}^{(2)}(t)$ for shared rooms. As medical costs usually evolve differently in shared rooms compared to private rooms, the medical inflation market index that can be used for the insurance product under consideration might be not appropriate. On the other hand, in case the claim payments related to the insurance product reveal proportions $51 \%$ / $49 \%$, the medical index that can be applied is the private room medical index, which might again not correspond to the medical inflation observed in the portfolio under consideration.

In the following example, we illustrate the calculation of medical inflation indexes according to the current Belgian approach.

Example 1 Consider a market with 4 lifelong health insurance products $(J=4)$ and two types of covers $(K=2)$ at time 1 . The claim amounts and number of policyholders observed in the previous two periods are given in Table 1.

\begin{tabular}{c|ccc|ccc|ccc|ccc}
\hline & \multicolumn{3}{|c|}{ Product 1 } & \multicolumn{3}{c|}{ Product 2 } & \multicolumn{3}{c|}{ Product 3 } & \multicolumn{3}{c}{ Product 4 } \\
$t$ & $C_{1}^{(1)}(t)$ & $C_{1}^{(2)}(t)$ & $l_{1}(t)$ & $C_{2}^{(1)}(t)$ & $C_{2}^{(2)}(t)$ & $l_{2}(t)$ & $C_{3}^{(1)}(t)$ & $C_{3}^{(2)}(t)$ & $l_{3}(t)$ & $C_{4}^{(1)}(t)$ & $C_{4}^{(2)}(t)$ & $l_{4}(t)$ \\
\hline 0 & 400 & 600 & 10 & 900 & 600 & 15 & 1800 & 0 & 30 & 0 & 2400 & 60 \\
1 & 880 & 1260 & 20 & 1800 & 1000 & 25 & 3600 & 0 & 50 & 0 & 4000 & 100 \\
\hline
\end{tabular}

Table 1: Claims and number of insureds for the market in Example 1.

In a first step, each product available in the market is classified as either a 'type of cover 1' or 'type of cover 2'- product, based on the type of cover of the product that leads to the largest proportion of claim payments. In this example we find for both observation years that products 2 and 3 are 'type of cover 1' - products, while products 1 and 4 are 'type of cover 2' - products.

Next, the medical inflation indexes of the market for both types of covers is determined 
according to (1):

$$
\frac{(900+600)+1800}{15+30} \times\left(1+i_{m}^{(1)}(1)\right)=\frac{(1800+1000)+3600}{25+50}
$$

and

$$
\frac{(400+600)+2400}{10+60} \times\left(1+i_{m}^{(2)}(1)\right)=\frac{(880+1260)+4000}{20+100} .
$$

This leads to

$$
i_{m}^{(1)}(1)=16.4 \% \text { and } i_{m}^{(2)}(2)=5.3 \% .
$$

The index $i_{m}^{(1)}(1)$ has to be applied to products 2 and 3 , while the premiums of products 1 and 4 are updated using the index $i_{m}^{(2)}(1)$ in the coming year $(1,2)$. Hence, $i_{2}(1)=i_{3}(1)=$ $16.4 \%$, while $i_{1}(1)=i_{4}(1)=5.3 \%$.

\section{Product-specific experience-based medical inflation indexes}

Before introducing an alternative way of calculating market-based indexes for all types of covers offered in the market, we first introduce product-specific experience-based indexes for each type of cover offered in the product under consideration. These indexes are constructed such that they capture the experienced health claim increases which are specific to the product and to the type of cover. We introduce these indexes to study in the subsequent section the accuracy of the newly proposed indexes with respect to the current Belgian ones.

The product-specific experience-based index for product $j$ and type of cover $k$ observed in year $(t-1, t)$ is denoted by $e_{j}^{(k)}(t)$. It is defined by

$$
\frac{C_{j}^{(k)}(t-1)}{l_{j}(t-1)}\left(1+e_{j}^{(k)}(t)\right)=\frac{C_{j}^{(k)}(t)}{l_{j}(t)}, \quad \text { for } j \in I^{(k)} .
$$

The factor $\left(1+e_{j}^{(k)}(t)\right)$ in 33 connects the 'type of cover $k$ ' - claim cost per insured of product $j$, over two consecutive years. Furthermore, in case $j \notin I^{(k)}$, we set $e_{j}^{(k)}(t)$ equal to 0 , by convention.

The overall product-specific index for product $j$ in year $(t-1, t)$ is denoted by $e_{j}(t)$. It follows from:

$$
\frac{C_{j}(t-1)}{l_{j}(t-1)}\left(1+e_{j}(t)\right)=\frac{C_{j}(t)}{l_{j}(t)} .
$$

Here, the factor $\left(1+e_{j}(t)\right)$ connects claim costs per insured for product $j$, over two consecutive years. 
It is straightforward to prove the following relation between the 'type of cover $k$ ' indexes $e_{j}^{(k)}(t)$ and the overall index $e_{j}(t)$ of the product:

$$
1+e_{j}(t)=\sum_{k=1}^{K}\left(1+e_{j}^{(k)}(t)\right) \times w_{j}^{(k)}(t)
$$

with weights $w_{j}^{(k)}(t)$ given by

$$
w_{j}^{(k)}(t)=\frac{C_{j}^{(k)}(t-1)}{C_{j}(t-1)}
$$

The product-specific index $e_{j}(t)$ can be defined for gross as well as net claim amounts. The index has the advantage of enabling the insurer to account for product-specific systematic risk (also called undiversifiable risk) due to medical inflation. However, it could also capture unsystematic (also called diversifiable or idiosyncratic) risk, which is supposed to be borne by the insurer, and hence should not trigger the medical indexing mechanism. Another disadvantage of applying this index for updating premiums is that it reflects the (gross or net) claim increases that were experienced by the insurer, which could constitute competitive sensitive information. Finally, using product-specific indexes $e_{j}(t)$ 's could lead to a lack of transparency toward the clients, and would not lead to uniform premium increases in the market.

\section{A new class of medical inflation indexes}

In this section, we propose a new method to construct medical inflation market indexes for the different types of covers, as well as for the product-specific indexes. In the construction of the index for type of cover $k$, we take into account all products offering that cover. In the application of the index for product $j$, we take into account all types of covers included in that product. Thus, compared to the current Belgian indexes, the proposed method provides market indexes which are more representative of the experienced market medical inflation for each type of cover, and allows for an indexing at the product-level which captures more accurately the medical inflation for that product.

In a first step, we define $f_{m}^{(k)}(t)$, the time- $t$ medical index of the market for type of cover $k$, as follows:

$$
\frac{\sum_{j \in I^{(k)}} C_{j}^{(k)}(t-1)}{\sum_{j \in I^{(k)}} l_{j}(t-1)} \times\left(1+f_{m}^{(k)}(t)\right)=\frac{\sum_{j \in I^{(k)}} C_{j}^{(k)}(t)}{\sum_{j \in I^{(k)}} l_{j}(t)} .
$$

Our previous assumptions ensures that $f_{m}^{(k)}(t)$ is well-defined for each type of cover $k$. The right hand side of this equation is the total market claims paid for 'type of cover $k$ ' in year $(t-1, t)$, divided by the total number of insurance contracts offering that cover. On the left hand side, the same average appears for the previous year $(t-2, t-1)$. The factor $\left(1+f_{m}^{(k)}(t)\right)$ connects both average claim amounts. 
In a second step, we propose that product $j$ is appointed a product-specific index, based on the relative importance of the different medical services covered by that product. Inspired by formula (5), we suggest the following calculation:

$$
1+f_{j}(t)=\sum_{k=1}^{K}\left(1+f_{m}^{(k)}(t)\right) \times w_{j}^{(k)}(t),
$$

with the weights defined by (6).

As opposed to the market indexes $i_{m}^{(k)}(t)$ defined in 11$)$, the market indexes $f_{m}^{(k)}(t)$ defined above are not biased by the claim amounts of other categories that do not contribute to the claim increases of category $k$. Moreover, the product-specific index $f_{j}(t)$ takes into account the weight of the type of cover in the total claims of the product. Therefore, it is to be expected that the newly proposed method will give rise to a more accurate indexing mechanism than the one currently used in Belgium.

Comparing formulas (5) and (8), we see that in the definition of $e_{j}(t)$, the type-of-coverrelated indexes $e_{j}^{(k)}(t)$ are used, whereas in the definition of $f_{j}(t)$, the type-of-cover-related indexes $f_{m}^{(k)}(t)$ are proposed. Calculation of the indexes $e_{j}^{(k)}(t)$ is performed at the product level, whereas the $f_{m}^{(k)}(t)$ are determined at the market level. Hence, $e_{j}(t)$ is derived from product-specific performance of the claims related to the covered type of medical service, whereas for $f_{j}(t)$ market averages are used for it. As the market portfolio is a larger pool than the portfolio of an individual product, the index $f_{j}(t)$ will be superior to the index $e_{j}(t)$ in terms of capturing the medical inflation due to systematic risk.

Concerning the claim amounts $C_{j}^{(k)}$ in formulas (6), (7) and (8), both the 'gross claims' and the 'net claims' interpretation are possible. In case the medical indexes $f^{(k)}(t), f_{j}(t)$ and the weights $w_{j}^{(k)}(t)$ are based on gross claims, the market indexes $f^{(k)}(t)$ capture the increase of gross claims in the market, while the weights $w_{j}^{(k)}(t)$ might not correctly capture the relative weights of the different types of covers in the product under consideration, leading to a wrong figure for the medical inflation $f_{j}(t)$. On the other hand, using the net claims to define the medical indexes and the weights, is an appropriate approach for the weights $w_{j}^{(k)}(t)$, but might give a wrong picture of the medical inflation indexes $f^{(k)}(t)$ for the different types of covered medical services in the market. One possible rule of thumb consists in determining the market indexes $f^{(k)}(t)$ using the formulas $(7)$, with the $C_{j}^{(k)}$ defined as gross claims, while the product-specific indexes $f_{j}(t)$ and the weights $w_{j}^{(k)}(t)$ are determined according to (6) and (8), with the $C_{j}^{(k)}$ interpreted as net claims.

Hereafter, we will numerically illustrate the validity of our approach. In the examples that we will consider, we always assume that all products have an unlimited cover, i.e. that gross claims and net claims are identical. Throughout the remaining numerical illustrations, we use the product-specific experience-based indexes (5) as benchmark. In the next example, we revisit the market considered in Example 1 and compare its indexes $i_{j}(1), e_{j}(1)$ and $f_{j}(1)$. 
Example 2 Consider the market with 4 products and 2 types of covers observed at time 1, as described in Table 1 of Example 1. The experience-based indexes for each product follow directly from (4), such that:

$$
\begin{aligned}
\frac{400+600}{10} \times\left(1+e_{1}(1)\right) & =\frac{880+1260}{20}, \\
\frac{900+600}{15} \times\left(1+e_{2}(1)\right) & =\frac{1800+1000}{25}, \\
\frac{1800}{30} \times\left(1+e_{3}(1)\right) & =\frac{3600}{50}, \\
\frac{2400}{60} \times\left(1+e_{4}(1)\right) & =\frac{4000}{100},
\end{aligned}
$$

and we find $e_{1}(1)=7 \%, e_{2}(1)=12 \%, e_{3}(1)=20 \%$ and $e_{4}(1)=0 \%$. For the proposed index $f_{j}$ of product $j$, the calculation is carried out in two steps. The first step is to determine the market medical indexes for each type of cover, i.e. the indexes $f_{m}^{(k)}$ defined in (7). For type of cover 1, we have:

$$
\left.\frac{400+900+1800}{10+15+30}\left(1+f_{m}^{(} 1\right)(1)\right) \frac{880+1800+3600}{20+25+50},
$$

which leads to $f_{m}^{1}(1) \approx 17.28 \%$. For type of cover 2 , we have:

$$
\left.\frac{600+600+2400}{10+15+60}\left(1+f_{m}^{(} 2\right)(1)\right) \frac{1260+1000+4000}{20+25+100},
$$

which leads to $f_{m}^{2}(1) \approx 1.93 \%$. We clearly see that medical inflation is much higher for type of cover 1 compared to that of type of cover 2 . The second step is to determine to medical inflation for each product $j$ using the weighted sum in (8). The couple of weights $\left(w_{j}^{(1)}(1), w_{j}^{(2)}\right)$ for each product $j=1,2,3$ and 4 is given by $(0.4,0.6),(0.6,0.4),(1,0)$ and $(0,1)$, respectively. Therefore, we find:

$$
\begin{aligned}
& 1+f_{1}(1) \approx(1+17.28 \%) \times 0.4+(1+1.93 \%) \times 0.6, \\
& 1+f_{2}(1) \approx(1+17.28 \%) \times 0.6+(1+1.93 \%) \times 0.4, \\
& 1+f_{3}(1) \approx(1+17.28 \%) \times 1+(1+1.93 \%) \times 0, \\
& 1+f_{4}(1) \approx(1+17.28 \%) \times 0+(1+1.93 \%) \times 1 .
\end{aligned}
$$

The indexes $i_{j}(1), e_{j}(1)$ and $f_{j}(1)$ are reported for all products in Table 2

In this market, the proposed indexes $f_{j}(1)$ provide a good approximation for the experience-based indexes $e_{j}(1)$, whereas the Belgian indexes $i_{j}(1)$ perform worse. This phenomenon is in particular observed for the single cover - product 4, which did not experience any medical inflation. For this product, the current Belgian system suggests to update the premiums based on a medical inflation of $i_{4}(1)=5.3 \%$, whereas our approach leads to $f_{4}(1)=1.9 \%$, which is much closer to the experience-based inflation $e_{4}(1)=0 . \nabla$

In the following example, we consider another market to illustrate the performance of the different medical inflation indexes. 


\begin{tabular}{r|cccc}
\hline & Product 1 & Product 2 & Product 3 & Product 4 \\
\hline Belgian indexes $i_{j}(1)$ & $5.3 \%$ & $16.4 \%$ & $16.4 \%$ & $5.3 \%$ \\
Proposed indexes $f_{j}(1)$ & $8.1 \%$ & $11.1 \%$ & $17.3 \%$ & $1.9 \%$ \\
Experience-based indexes $e_{j}(1)$ & $7.0 \%$ & $12.0 \%$ & $20.0 \%$ & $0.0 \%$ \\
\hline
\end{tabular}

Table 2: Comparison of the different medical indexes for the market of Example1.

Example 3 Consider a market with 4 products $(J=4)$ and 2 types of covers $(K=2)$. Each product's claim amounts for the different types of covers and number of policies in two consecutive years are displayed in Table 3 .

\begin{tabular}{c|ccc|ccc|ccc|ccc}
\hline & \multicolumn{3}{|c|}{ Product 1 } & \multicolumn{3}{|c|}{ Product 2 } & \multicolumn{3}{c|}{ Product 3 } & \multicolumn{3}{c}{ Product 4 } \\
$t$ & $C_{1}^{(1)}(t)$ & $C_{1}^{(2)}(t)$ & $l_{1}(t)$ & $C_{2}^{(1)}(t)$ & $C_{2}^{(2)}(t)$ & $l_{2}(t)$ & $C_{3}^{(1)}(t)$ & $C_{3}^{(2)}(t)$ & $l_{3}(t)$ & $C_{4}^{(1)}(t)$ & $C_{4}^{(2)}(t)$ & $l_{4}(t)$ \\
\hline 0 & 400 & 600 & 10 & 900 & 600 & 15 & 1800 & 0 & 30 & 0 & 2000 & 50 \\
1 & 880 & 1500 & 20 & 1650 & 1250 & 25 & 3300 & 0 & 50 & 0 & 5000 & 100 \\
\hline
\end{tabular}

Table 3: Observed claims for the market of Example 3.

In this example, we find that all experience-based type-of-cover-specific indexes are equal, in the whole market:

$$
e_{j}^{(1)}(1)=10 \% \quad \text { and } \quad e_{j}^{(2)}(1)=25 \%, \quad \text { for } j=1,2 .
$$

Hence, the claim amounts per-policy for types of covers 1 and 2 increase by $10 \%$ and $25 \%$, respectively, for all products. The values of the indexes $i_{j}(1), e_{j}(1)$ and $f_{j}(1)$ are summarized in Table 4 . We observe again that the newly proposed index $f_{j}(1)$ outperforms the Belgian index $i_{j}(1)$, when compared to the experience-based index $e_{j}(1)$.

\begin{tabular}{r|cccc}
\hline & Product 1 & Product 2 & Product 3 & Product 4 \\
\hline Belgian indexes $i_{j}(1)$ & $23.0 \%$ & $12.7 \%$ & $12.7 \%$ & $23.0 \%$ \\
Proposed indexes $f_{j}(1)$ & $18.7 \%$ & $15.4 \%$ & $8.9 \%$ & $25.3 \%$ \\
Experience-based indexes $e_{j}(1)$ & $19.0 \%$ & $16.0 \%$ & $10.0 \%$ & $25.0 \%$ \\
\hline
\end{tabular}

Table 4: Comparison of the medical indexes for the market in Example 3 .

In the (theoretical) special case of a market with a single product, i.e. $J=1$, the current Belgian index, the experience based index and the newly proposed index are identical:

$$
i_{1}(t)=e_{1}(t)=f_{1}(t) .
$$

The proof of these equalities follows in a straightforward way from (1), (5) and (8). In the following theorem, we move to the more realistic case of a multiple product market. We consider conditions under which the product-specific index $e_{j}(t)$ and our newly proposed index $f_{j}(t)$ are equal. 
Theorem 1 Consider a market at time $t$ and suppose that in the period $(t-1, t)$, the observed average claims for any given type of cover are equal for all products which include that cover, i.e.

$$
\frac{C_{j}^{(k)}(t)}{l_{j}(t)}=c^{(k)}(t), \quad \text { for any } k \in\{1,2, \ldots, K\} \text { and } j \in I^{(k)} .
$$

Furthermore, suppose that the corresponding observation also holds for the period $(t-2, t-1)$ :

$$
\frac{C_{j}^{(k)}(t-1)}{l_{j}(t-1)}=c^{(k)}(t-1), \quad \text { for any } k \in\{1,2, \ldots, K\} \text { and } j \in I^{(k)} .
$$

Then for any product $j$ in the market, the indexes $e_{j}(t)$ and $f_{j}(t)$ are equal:

$$
e_{j}(t)=f_{j}(t), \quad \text { for any } j=1,2, \ldots, J .
$$

Proof: Taking into account (9) and (10), we immediately find from (3) that

$$
1+e_{j}^{(k)}(t)=\frac{c^{(k)}(t)}{c^{(k)}(t-1)}, \quad \text { for any } k \in\{1,2, \ldots, K\} \text { and } j \in I^{(k)} .
$$

Hence, for any product $j$ which includes type of cover $k$, the index $e_{j}^{(k)}(t)$ is independent of $j$. From (7), (9), 10) and (12) it follows then that

$$
e_{j}^{(k)}(t)=f_{m}^{(k)}(t), \quad \text { for any } k \text { in }\{1,2, \ldots, K\} \text { and } j \in I^{(k)} .
$$

Observing that $w_{j}^{(k)}(t)=0$ in case $j \notin I^{(k)}$ and comparing definitions (5) and (8) of the indexes $f_{j}(t)$ and $e_{j}(t)$, the equalities derived above lead to (11).

Important to notice is that although conditions $(9)$ and $(10)$ imply the equality of the indexes $f_{j}(t)$ and $e_{j}(t)$ for any product $j$, in general these conditions do not imply that also the indexes $i_{j}(t)$ and $e_{j}(t)$ are equal. This phenomenon is illustrated in the following example.

Example 4 At time 1, we consider a market with 2 products and 2 types of covers of which the claim amounts in the previous two periods are given in Table 5. Product 1 only includes type of cover 1 , whereas product 2 offers both types of covers. Therefore, we have $I^{(1)}=\{1,2\}$ and $I^{(2)}=\{2\}$. Furthermore, from Table 5, we find that $M^{(1)}(t)=\{1,2\}$ for $t=0$ and 1 , whereas $M^{(2)}(0)$ and $M^{(2)}(1)$ are empty sets. One can easily verify that in this market, conditions (9) and (10) of Theorem 1 are fulfilled.

The indexes for this market are reported in Table 6. Clearly, the Belgian indexes fail to properly capture the experienced medical inflation in this case. In particular, the Belgian indexes $i_{1}(1)$ and $i_{2}(1)$ are equal, while the other indexes lead to different values for both products. 


\begin{tabular}{c|ccc|ccc}
\hline & \multicolumn{3}{|c|}{ Product 1 } & \multicolumn{3}{c}{ Product 2 } \\
$t$ & $C_{1}^{(1)}(t)$ & $C_{1}^{(2)}(t)$ & $l_{1}(t)$ & $C_{2}^{(1)}(t)$ & $C_{2}^{(2)}(t)$ & $l_{2}(t)$ \\
\hline 0 & 100 & 0 & 10 & 200 & 150 & 20 \\
1 & 120 & 0 & 10 & 240 & 200 & 20 \\
\hline
\end{tabular}

Table 5: Observed claims for the market of Example 4.

\begin{tabular}{r|cc}
\hline & Product 1 & Product 2 \\
\hline Belgian indexes $i_{j}(1)$ & $24.4 \%$ & $24.4 \%$ \\
Proposed indexes $f_{j}(1)$ & $20.0 \%$ & $25.7 \%$ \\
Experience-based indexes $e_{j}(1)$ & $20.0 \%$ & $25.7 \%$ \\
\hline
\end{tabular}

Table 6: Comparison of the medical indexes for the market in Example 4.

The example above illustrates the fact that the conditions of Theorem 1 are not sufficient to ensure that the Belgian indexes $i_{j}(t)$ of the different products are equal to the respective product-specific indexes $e_{j}(t)$. This has to be considered as a weakness of the current Belgian medical inflation indexes. Our newly introduced indexes $f_{j}(t)$ do not exhibit this weakness.

Finally, consider a product $j$ for which some of the experience-based indexes $e_{j}^{(k)}(t)$ are very different from the newly introduced indexes $f_{m}^{(k)}(t)$. For such a product also the global experience-based index $e_{j}(t)$ might be very different from the newly introduced index $f_{j}(t)$, indicating that product $j$ is very different from the average product in the market. In this case, the product might still need an approach different from the one mentioned above in order to restore actuarial equivalence. Note that Belgian law allows for a personalized updating upon approval from the regulating authority.

\section{Concluding remarks}

Portfolios of lifelong health insurance contracts are subject to systematic medical inflation risk. In order to cope with this unpredictable risk, the Belgian regulation allows private and mutual insurers to update level premiums of lifelong health insurance contracts, using specific medical inflation indexes, which are based on aggregated market data. The construction of these indexes remains a challenge. Although such medical inflation indexes are so far a Belgian originality, as pointed out in Devolder et al. (2008), their construction remains relevant to other countries, provided there is a sufficient number of contracts with lifelong cover and potential adjustments of the premiums during the life of the contract. One example of countries for which this is the case is Germany; see Schneider (2002). Another example is the Indian health insurance market where some contracts also provide a lifelong health cover. Moreover, these indexes can also be useful in other applications such as the study of the drivers of medical inflation in a governmental context. 
In the present note, we proposed a new methodology for the calculation of these indexes when different products with multiple types of covers are sold in the market. We compare the accuracy of the newly proposed indexes with the current Belgian approach on the basis of some numerical (theoretical) examples, where company-specific experiencebased indexes are taken as benchmark. Although the latter indexes allow a tailor-made updating for each product sold in the market, their application might be not desirable due to several reasons which were discussed above. The newly proposed medical indexes can be considered as improved versions of the current indexes prescribed by the Belgian regulator, as they better reflect the experienced medical inflation of any particular health insurance portfolio.

Hereafter, we address some practical issues related to defining medical inflation indexes which are not covered in the present paper.

The experience-based index $e_{j}^{(k)}(t)$ introduced in $(5)$ can be expressed as a function of an experience-based medical frequency index and an experience-based medical severity index. Let us introduce the notation $d_{j}^{(k)}(t)$ for the number of insureds of product $j$ in year $(t-1, t)$ with a 'type of cover - $k$ ' claim in that year. The medical frequency index $n_{j}^{(k)}$ for product $j$ and category $k$ is defined by

$$
\frac{d_{j}^{(k)}(t-1)}{l_{j}(t-1)}\left(1+n_{j}^{(k)}(t)\right)=\frac{d_{j}^{(k)}(t)}{l_{j}(t)},
$$

while the corresponding medical severity index $y_{j}^{(k)}$ follows from

$$
\frac{C_{j}^{(k)}(t-1)}{d_{j}^{(k)}(t-1)}\left(1+y_{j}^{(k)}(t)\right)=\frac{C_{j}^{(k)}(t)}{d_{j}^{(k)}(t)}
$$

Obviously, the medical frequency index measures the 'frequency inflation' in two consecutive years, whereas the medical severity index measures the 'severity inflation'. From (4), 13 and 14 , we find the following relation between the experience-based indexes $e_{j}^{(k)}(t)$, $n_{j}^{(k)}(t)$ and $y_{j}^{(k)}(t)$ of product $j$ and category $k$ :

$$
\left(1+e_{j}^{(k)}(t)\right)=\left(1+n_{j}^{(k)}(t)\right)\left(1+y_{j}^{(k)}(t)\right) .
$$

This decomposition of the medical inflation in a frequency and a severity component can be useful to analyze the drivers of medical inflation. However, such an analysis that could build on earlier work of e.g. Bachler et al. (2006) is out of the scope of the current paper.

A relevant issue when determining market-wide medical inflation indexes, which is not considered in this paper, is how to take into account waiting periods, which are typical for newly underwritten contracts, in the calculation of the medical index. This problem could arise e.g. in case the market experiences a substantial growth in contracts. Not carefully taking into account the waiting period may lead to medical inflation indexes which are not appropriate. One simple solution consists of not taking into account policies which are still in the waiting period when calculating the medical indexes. 
Acknowledgment: The authors acknowledge the financial support of AG Insurance in the framework of the $A G$ Insurance Chair In Health Insurance at KU Leuven. The authors are also grateful to Prof. Michel Denuit from UC Louvain and Prof. Julien Trufin from Université Libre de Bruxelles, as well as to two anonymous referees for their valuable comments.

\section{References}

Bachler, R., Duncan, I. and Juster, I. (2006), 'A comparative analysis of chronic and nonchronic insured commercial member cost trends', North American Actuarial Journal 10, 76-89.

Denuit, M., Dhaene, J., Hanbali, H., Lucas, N. and Trufin, J. (2017), 'Updating mechanism for lifelong insurance contracts subject to medical inflation', European Actuarial Journal (7), 133-163.

Devolder, P., Denuit, M., Maréchal, X., Yerna, B.-L., Closon, J.-P., Léonard, C., Senn, A. and Vinck, I. (2008), 'Construction d'un index médical pour les contrats privés d'assurance maladie', KCE Reports - Centre Fédéral d'Expertise des Soins de Santé (96B).

Dhaene, J., Godecharle, E., Antonio, K., Denuit, M. and Hanbali, H. (2017), 'Lifelong health insurance covers with surrender values: updating mechanisms in the presence of medical inflation', ASTIN Bulletin (47), 803-836.

Hanbali, H., Claassens, H., Denuit, M., Dhaene, J. and Trufin, J. (2017), 'Application de l'indice médical dans les contrats d'assurance maladie en Belgique', Research report AFI-17114, KU Leuven .

Schneider, E. (2002), 'The main features of German private health insurance', Preceedings of the 27th ICA Health Seminar, Cacun . 